\title{
Isolation and characterization of duck adenovirus 3 circulating in China
}

\author{
Shaohua Shi ${ }^{1,2,3} \cdot$ Rongchang Liu ${ }^{1,2,3} \cdot$ Chunhe Wan ${ }^{1,2,3} \cdot$ Longfei Cheng $^{1,2,3} \cdot$ Zhen Chen $^{1,2,3} \cdot$ Guanghua Fu ${ }^{1,2,3}$. \\ Hongmei Chen ${ }^{1,2,3}$. Qiuling Fu ${ }^{1,2,3} \cdot$ Yu Huang ${ }^{1,2}$
}

Received: 26 August 2018 / Accepted: 5 November 2018 / Published online: 18 December 2018

(c) The Author(s) 2018

\begin{abstract}
Recently, infectious disease outbreaks characterized by swelling and hemorrhagic liver and kidneys occurred in Muscovy ducklings in China. Four viruses were isolated and identified as adenoviruses by transmission electron microscopy (TEM) and polymerase chain reaction (PCR). Sequence analysis identified the new isolates as duck adenovirus 3 (DAdV-3), species Duck aviadenovirus $B$. The pathogenicity of the new isolate DAdV-3 FJGT01 was investigated using challenge experiments. The gross lesions in the animal experiment were similar to the clinical lesions observed in the diseased ducks. TEM examination of liver sample showed that virions accumulated and arranged in crystal lattice formations in the nuclei of hepatocytes. The present study provides new information about the epidemiology and characteristics of duck adenovirus associated with Muscovy ducklings.
\end{abstract}

\section{Introduction}

According to the International Committee on Taxonomy of Viruses (ICTV), the family Adenoviridae includes five genera: Atadenovirus, Aviadenovirus, Ichtadenovirus, Mastadenovirus and Siadenovirus [1]. The genus Aviadenovirus includes 14 species: Fowl aviadenovirus A-E and Goose aviadenovirus $A$, Duck aviadenovirus $B$, Pigeon aviadenovirus $A-B$, Falcon aviadenovirus A, Psittacine aviadenovirus $B$,

Handling Editor: T. K. Frey.

Shaohua Shi and Rongchang Liu have contributed equally to this work.

Electronic supplementary material The online version of this article (https://doi.org/10.1007/s00705-018-4105-2) contains supplementary material, which is available to authorized users.

Yu Huang

huangyu_815@163.com

1 Institute of Animal Husbandry and Veterinary, Fujian Academy of Agricultural Sciences, 247 Wusi Road, Fuzhou, Fujian, People's Republic of China

2 Fujian Provincial Key Laboratory for Avian Diseases Control and Prevention, Fuzhou, Fujian, People's Republic of China

3 Fujian Animal Diseases Control Technology Development Center, Fuzhou, Fujian, People's Republic of China and Turkey aviadenovirus $B$ - $D$ [2]. So far, two duck adenoviruses (DAdVs) have been classified by the ICTV: DAdV-1 in the genus Atadenovirus and DAdV-2 in the genus Aviadenovirus. Waterfowl are known to be natural hosts of DAdV-1, and infections are usually asymptomatic [3]. In chickens and quail, DAdV-1 has been described as the causative agent of egg production problems $[4,5]$. To our knowledge, infection with DAdV-2 has only been reported in ducklings. In 2014, Marek et al. published the complete genomic sequence of DAdV-2 GR isolated from Muscovy ducklings in France. Based on its genetic characteristics, DAdV-2 has been suggested to belong to the genus Aviadenovirus and the species Duck aviadenovirus $B$ [6]. Another duck adenovirus was isolated almost simultaneously from Muscovy ducklings in China and then designated as a DAdV-3 candidate [7].

Recently, several emerging outbreaks, characterized by swelling and hemorrhagic liver and kidney, have been threatening Muscovy duckling farms in Fujian, Zhejiang, Anhui and Guangdong provinces in China (Fig. 1). The morbidity has ranged from $40 \%$ to $55 \%$, with a mortality rate of $35 \%-43 \%$. To identify the causative agents, liver samples were collected from the affected Muscovy ducklings. Due to suspicion of possible bacterial infections, each sample was subjected to bacterial isolation using blood agar plates and tryptic soy agar plates as described by Liu et al. [8]. None of the samples were positive for bacterial isolation. The remaining samples were homogenized in Dulbecco's 
Fig. 1 Gross lesion on the liver (A) and the kidney (B) of dead ducklings showing swelling and hemorrhage
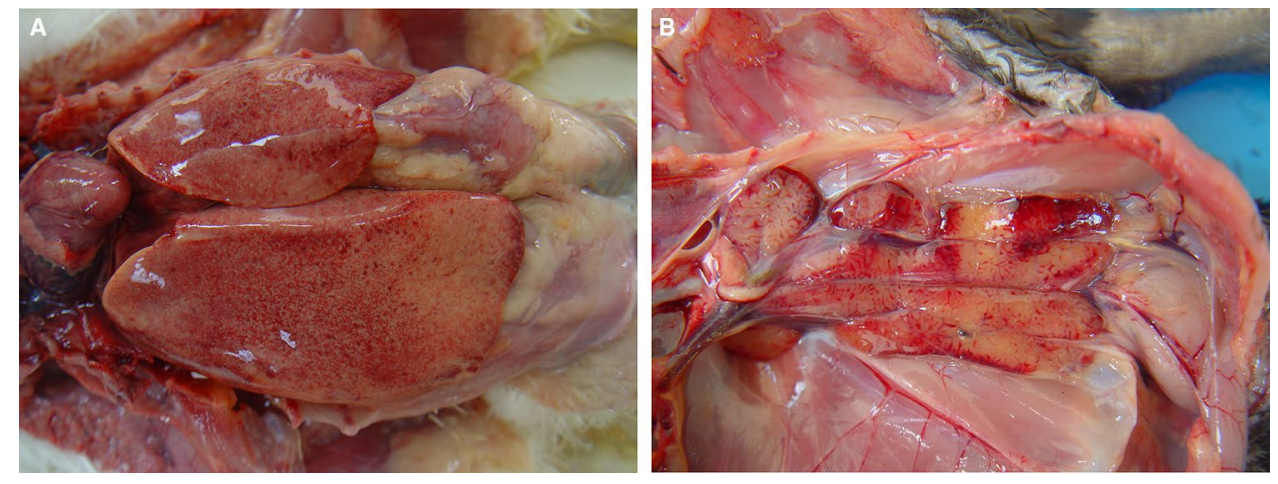

modified Eagle's medium (DMEM, Gibco, Grand Island, NY, USA). The tissue suspensions were subjected to three rounds of freeze-thawing before centrifugation at $5,000 \mathrm{~g}$ for $15 \mathrm{~min}$ at $4{ }^{\circ} \mathrm{C}$. The supernatants were then used for polymerase chain reaction (PCR) or reverse transcription PCR (RT-PCR) assays to detect avian influenza virus [9], avian paramyxovirus type 1, duck virus hepatitis A types 1 and 3 [10, 11], Muscovy duck reovirus [12], novel duck reovirus [13], avian Tembusu virus [14], duck astroviruses [15], Muscovy duck parvovirus [16], goose parvovirus [16], duck enteritis virus [17] and aviadenovirus (AdV) [18]. The samples were positive only for AdV.

Duck embryo fibroblasts (DEFs) were freshly prepared from 11-day-old Muscovy duck embryos and maintained in DMEM supplemented with 5\% fetal calf serum (FCS, Gibco, Grand Island, NY, USA) for $\sim 16$ hours. AdV-positive samples were inoculated onto DEFs, and four virus isolates, designated as FJGT01, AHAQ13, ZJJH07 and GDMM10, were isolated successfully. Compared to normal DEFs, the infected cells became round and clustered like grapes (Fig. S1), which is a classical characteristic of the cytopathic effect in adenovirus-infected cells [19-22]. The titers of FJGT01, AHAQ13, ZJJH07 and GDMM10 were determined as $10^{5.5}$ TCID50/ml, $10^{3.5}$ TCID50/ml, $10^{4.3}$ TCID50/ml, $10^{3.0} \mathrm{TCID} 50 / \mathrm{ml}$, respectively. For transmission electron microscopy (TEM) examination, the cell culture fluid of infected DEFs was harvested and then clarified by centrifugation at $8,000 \mathrm{~g}$ for $30 \mathrm{~min}$ using a Ti19 rotor (Beckman Coulter, CA, USA) at $4{ }^{\circ} \mathrm{C}$. The supernatant was collected, overlaid onto a $20 \%$ sucrose solution, and then centrifuged at $100,000 \mathrm{~g}$ for $3 \mathrm{~h}$ at $4{ }^{\circ} \mathrm{C}$ in a Ti70 rotor (Beckman Coulter, CA, USA). The pellet was resuspended in TE buffer (10 mM Tris, pH 8.0, 1 mM EDTA, pH 8.0). The virus preparations were stained with phosphotungstic acid (Ted Pella Inc., Redding, CA, USA) and examined using TEM. Spherical particles ranging from $60 \mathrm{~nm}$ to $80 \mathrm{~nm}$ in diameter were observed (Fig. S2). These results indicated that the newly isolated viruses were AdVs.

The complete genomes of the identified were amplified using a set of specific primers (Table S1). PCR was carried out in a PCR system (C100 Touch ${ }^{\mathrm{TM}}$ Thermal Cycler, BioRad, Hercules, CA, USA). The PCR products were purified using a QIAquick Gel Extraction Kit (QIAGEN, Düsseldorf, Germany) and then cloned into pGEM®-T Easy Vector (Promega, Madison, WI, USA) according to the manufacturer's instructions. After transformation of competent Escherichia coli strain DH5 $\alpha$ (TaKaRa, Dalian, China) with these constructs, three positive clones for each PCR product were sequenced in both directions using an ABI 3730XL DNA Analyzer (Applied Biosystems, CA, USA) at BioSune Biotech Co., Shanghai, China. The genomic sequences of the $\mathrm{AdV}$ isolates were assembled using the Lasergene sequence analysis software package (DNASTAR Inc., WI, USA). The complete genomic sequences of FJGT01, AHAQ13, ZJJH07 and GDMM10 isolates were obtained and deposited in the GenBank database with the accession numbers MH777395, MH777396, MH777397 and MH777398, respectively. The full-length genomes of all four isolates were 43842 bp in length. The $\mathrm{G}+\mathrm{C}$ content of $47.13 \%$ and the predicted ORFs were similar to those reported for other adenoviruses.

Sequence comparisons showed that the newly isolated AdVs shared nearly $100 \%$ genomic sequence identity and $100 \%, 99.8 \%-100 \%$, and $100 \%$ amino acid identity in the hexon, fiber 1 , and fiber 2 proteins with the first DAdV-3 isolate CH-GD-12-2014. However, all four isolates shared only $86.9 \%$ amino acid sequence identity and $76.6 \%$ nucleotide sequence identity in the hexon with DAdV-2 GR. The most significant difference between the new isolates and DAdV-2 GR was that the newly isolated AdVs contained fiber 1 and fiber 2 genes, whereas the DAdV-2 GR isolate only had one fiber gene. These data suggested that the newly isolated AdVs were DAdV-3 strains.

Phylogenetic distance based primarily on distance matrix analysis of the DNA polymerase amino acid sequence is an important standard for the classification of aviadenoviruses according to the Ninth Report of the ICTV [1]. A new adenovirus species can be established if the phylogenetic distance of the DNA polymerase amino acid sequence ranges from $>5 \%$ to $15 \%$ in association with a lack of cross-neutralization if antisera are available $[1,23]$. The phylogenetic 
distance based on the DNA polymerase amino acid sequence between the newly isolated DAdV-3 strains and DAdV-2 GR, the representative strain of the species Duck aviadenovirus $B$, was nearly zero (0.004), suggesting that the new isolates DAdV-3 FJGT01, GDMM10, AHAQ13, and ZJJH07 are members of the species Duck aviadenovirus B (Fig. 2).

To verify the agent, animal experiments were carried out. All animal studies were done in compliance with the regulations and guidelines of Fujian Academy of Agricultural Sciences for institutional animal care (No.1306129FAAS). Briefly, a total of 30 ten-day-old Muscovy ducklings were randomly divided into two groups (15 ducklings per group). The ducklings in the infection group were inoculated with $300 \mu \mathrm{l}$ of FJGT01 containing $10^{5.5} \mathrm{TCID} 50 / \mathrm{ml}$ via the intramuscular route. Another group was inoculated intramuscularly with $300 \mu \mathrm{l}$ of phosphate-buffered saline as a control. All of the Muscovy ducklings were monitored for 14 days. In the infection group, FJGT01 was highly virulent for Muscovy ducklings, with a mortality rate of $60 \%$, which started on day 5 after inoculation. Gross lesions showed signs of swelling and hemorrhage in the liver and the kidneys of infected ducklings. All Muscovy ducklings in the control group remained clinically healthy.

Tissues of the heart, liver, spleen, lung, kidney, brain, and pancreas of dead Muscovy ducklings were collected for histological examination. The tissues were fixed, cut into sections, and stained with hematoxylin and eosin (Fig. 3). Histopathological examination of the treated group revealed congestion in the liver, and the dilated sinusoid was full of red blood cells. In addition, hepatocytes were swollen and denatured with round or irregular vacuoles that appeared in the cytoplasm. The myocardial fiber was edematous, coarser, and slightly congested. Congestion was also observed in the kidney. The inflammatory cells were aggregated. Renal tubular epithelial cells were swollen and degenerated, and some epithelial cells were necrotic. However, there were no obvious histological lesions in the spleen, lung, brain, or pancreas. No significant histopathological damage was found in ducklings from the control group.
Fig. 2 Phylogenetic analysis of duck adenoviruses and their counterparts based on the amino acid sequence of DNA polymerase. The tree was build using the neighbor-joining algorithm. Values at branches represent bootstrap support based on 1000 replicates. The new isolates are indicated by solid red circles

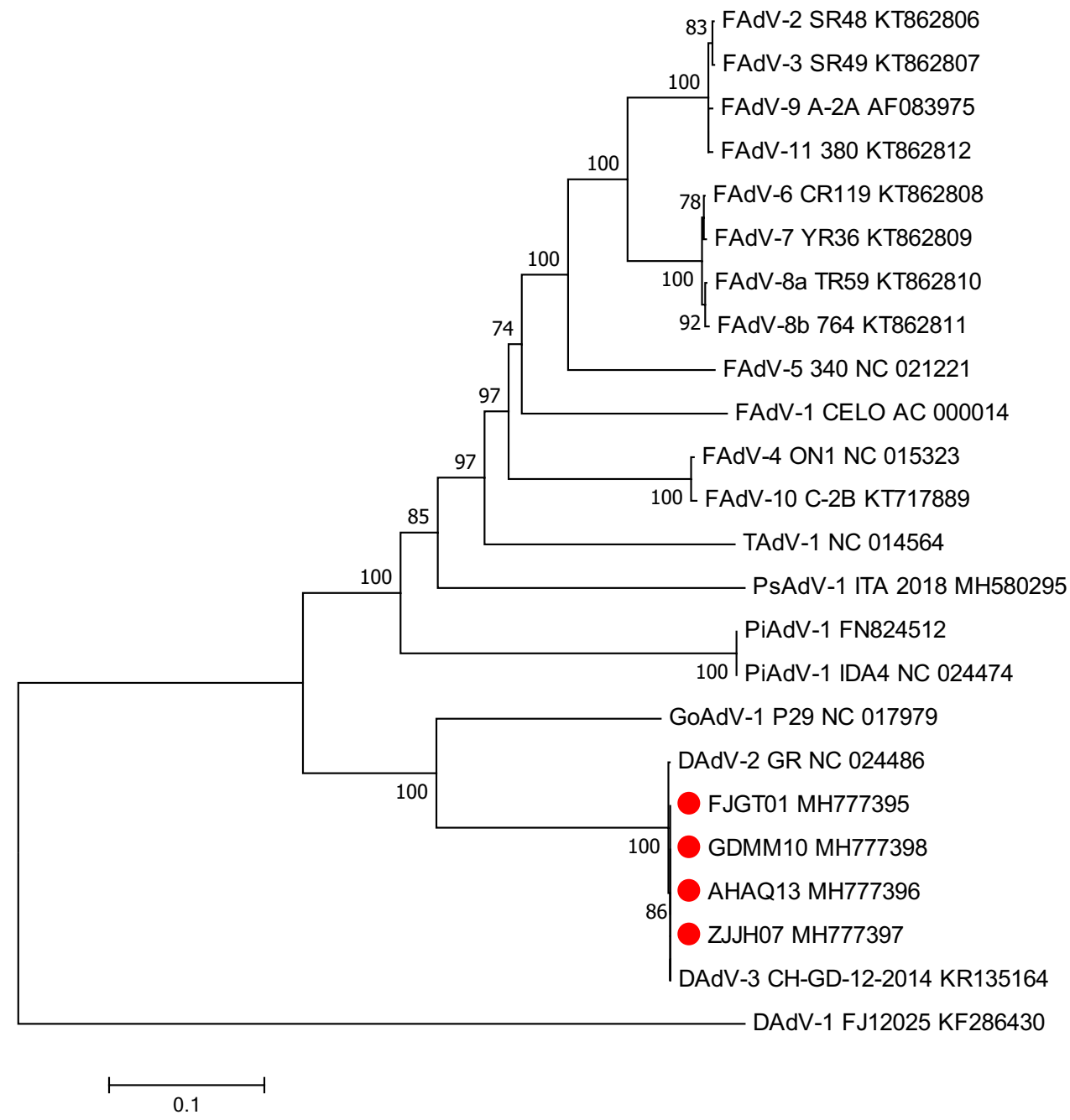


Fig. 3 Histopathological analysis of tissues from dead Muscovy ducklings infected with DAdV-3 FJGT01. The liver (A, infected group), kidney (C, infected group), and heart (E, infected group), showed obvious hemorrhage (black arrows). B, $\mathrm{D}$ and $\mathrm{E}$ show the histopathological observations in liver, kidney and heart, respectively, of Muscovy ducklings in the control group $($ scale $=50 \mu \mathrm{m})$
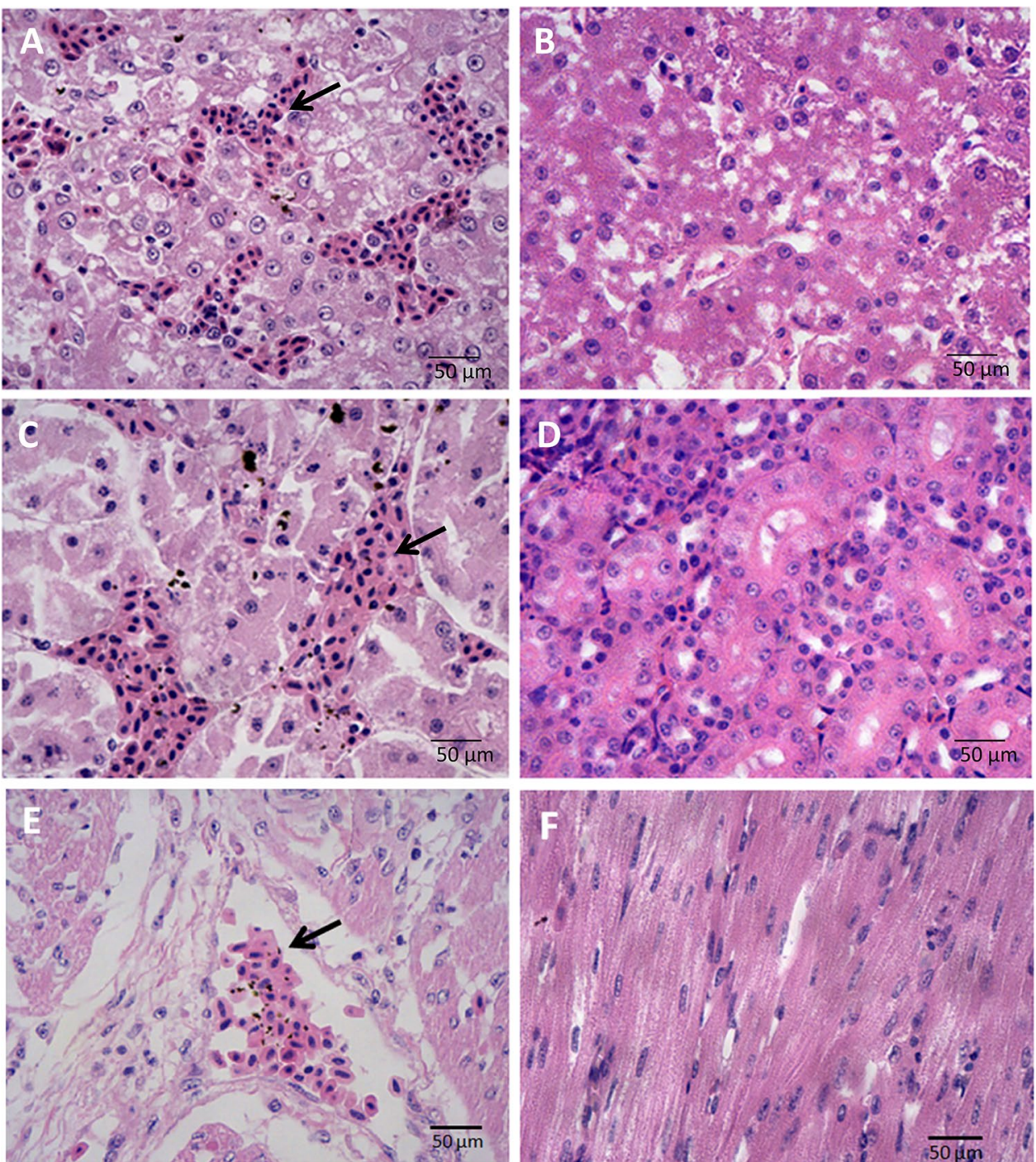

Liver samples were also collected for examination by TEM. The liver samples were embedded, and ultrathin sections were stained [24]. TEM demonstrated the presence of numerous icosahedral, nonenveloped viral particles measuring $60 \mathrm{~nm}$ to $80 \mathrm{~nm}$ in diameter. These virions were accumulated and arranged in crystal lattice formations in the nuclei of hepatocytes (Fig. 4), which is consistent with adenoviral infection [25-28]. This suggests that the liver is a tropic tissue of DAdV-3 and that viruses are synthesized and packaged in the nuclei of target cells. The structure, size, and lattice formation by these viral particles supported the diagnosis of an adenoviral infection.
Fig. 4 Electron microscopic view of the hepatic cell ultrastructure with accumulation of viral particles in the nucleus arranged in a crystal lattice (white arrow)
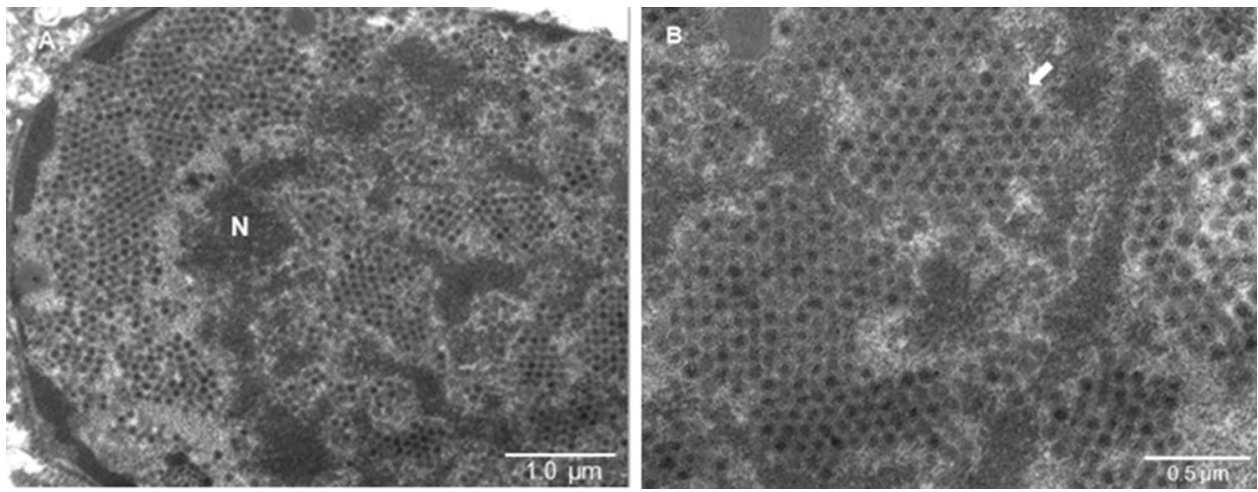
In summary, in the present study, we isolated and identified DAdV-3 that was circulating in China. The newly isolated DAdV-3 strains could be classified as numbers of the species Duck aviadenovirus B in the genus Aviadenovirus.

Funding This work was supported by the National Key Research and Development Program of China (Project no. 2017YFD0500802021), the National Natural Science Foundation of China (no. 31472222), Fund for Modern Agro-Industry Technology Research System (CARS42) from the Ministry of Agriculture of P. R. China, the Natural Science Foundation of Fujian Province (no. 2017J01060), the Basic Scientific Research Funds of Public Welfare Scientific Research Institutes of Fujian Province (2016R1022-1 and 2016R1022-9) and Freely explores scientific research project of FAAS (MNKZ 2018-248).

\section{Compliance with ethical standards}

Conflict of interest All authors declare that they have no conflict of interest.

Ethical approval All animal experiments were approved by the animal welfare committee of Fujian Academy of Agricultural Sciences (no. 1306129FAAS).

Open Access This article is distributed under the terms of the Creative Commons Attribution 4.0 International License (http://creativeco mmons.org/licenses/by/4.0/), which permits unrestricted use, distribution, and reproduction in any medium, provided you give appropriate credit to the original author(s) and the source, provide a link to the Creative Commons license, and indicate if changes were made.

\section{References}

1. Harrach B, Benkő M, Both GW et al (2011) Family adenoviridae. In: King A, Adams M, Lefkowitz E (eds) Virus taxonomy: classification and nomenclature of viruses. Ninth report of the international committee on taxonomy of viruses. Elsevier, San Diego, pp 25-41

2. Teske L, Rubbenstroth D, Meixner M et al (2017) Identification of a novel aviadenovirus, designated pigeon adenovirus 2 in domestic pigeons (Columba livia). Virus Res 227:15-22

3. Gulka CM, Piela TH, Yates VJ et al (1984) Evidence of exposure of waterfowl and other aquatic birds to the hemagglutinating duck adenovirus identical to EDS-76 virus. J Wildl Dis 20:1-5

4. Das BB, Pradhan HK (1992) Outbreaks of egg drop syndrome due to EDS-76 virus in quail (Coturnix coturnix japonica). Vet Rec 131:264-265

5. Van Eck JH, Davelaar FG, Heuvel-Plesman TA et al (1976) Dropped egg production, soft shelled and shell-less eggs associated with appearance of precipitins to adenovirus in flocks of laying fowls. Avian Pathol 5:261-272

6. Marek A, Kajan GL, Kosiol C et al (2014) Complete genome sequences of pigeon adenovirus 1 and duck adenovirus 2 extend the number of species within the genus Aviadenovirus. Virology 462-463:107-114

7. Zhang X, Zhong Y, Zhou Z et al (2016) Molecular characterization, phylogeny analysis and pathogenicity of a Muscovy duck adenovirus strain isolated in China in 2014. Virology 493:12-21

8. Liu R, Chen C, Huang Y et al (2018) Microbiological identification and analysis of waterfowl livers collected from backyard farms in southern China. J Vet Med Sci 80:667-671
9. Lee MS, Chang PC, Shien JH et al (2001) Identification and subtyping of avian influenza viruses by reverse transcription-PCR. J Virol Methods 97:13-22

10. Anchun C, Mingshu W, Hongyi X et al (2009) Development and application of a reverse transcriptase polymerase chain reaction to detect Chinese isolates of duck hepatitis virus type 1. J Microbiol Methods 77:332-336

11. Zhang R, Xia L, Chen J et al (2017) Molecular epidemiology and genetic diversity of duck hepatitis A virus type 3 in Shandong province of China, 2012-2014. Acta Virol 61:463-472

12. Yun T, Yu B, Ni Z et al (2013) Isolation and genomic characterization of a classical Muscovy duck reovirus isolated in Zhejiang, China. Infect Genet Evol 20:444-453

13. Ye WC, Yu B, Liu YS et al (2014) Establishment of universal RT-PCR for detection of Muscovy duck reovirus. Acta Agric Zhejiangensis 6:1453-1456

14. Wan CH, Shi SH, Cheng LF et al (2011) Establishment of RTPCR for detecting duck hemorrhagic ovaritis causing abrupt egglaying reduction in ducks. Fujian J Agric Sci 26:11-12

15. Todd D, Smyth VJ, Ball NW et al (2009) Identification of chicken enterovirus-like viruses, duck hepatitis virus type 2 and duck hepatitis virus type 3 as astroviruses. Avian Pathol 38:21-30

16. Liu JS, Jiang Q, Si CD et al (2007) Establishment of PCR assay for differentiation of Muscovy duck parvovirus from goose parvovirus. Vet Sci China 6:469-472

17. Hansen WR, Brown SE, Nashold SW et al (1999) Identification of duck plague virus by polymerase chain reaction. Avian Dis 43:106-115

18. Schrenzel M, Oaks JL, Rotstein D et al (2005) Characterization of a new species of adenovirus in falcons. J Clin Microbiol 43:3402-3413

19. Xie L, Yu XF, Sun Z et al (2012) Two adenovirus serotype 3 outbreaks associated with febrile respiratory disease and pharyngoconjunctival fever in children under 15 years of age in Hangzhou, China, during 2011. J Clin Microbiol 50:1879-1888

20. Liu Y, Wan W, Gao D et al (2016) Genetic characterization of novel fowl aviadenovirus 4 isolates from outbreaks of hepatitishydropericardium syndrome in broiler chickens in China. Emerg Microbes Infect 5:e117

21. Niczyporuk JS (2017) Molecular characterisation of fowl adenovirus type 7 isolated from poultry associated with inclusion body hepatitis in Poland. Arch Virol 162:1325-1333

22. Hematian A, Sadeghifard N, Mohebi R et al (2016) Traditional and modern cell culture in virus diagnosis. Osong Public Health Res Perspect 7:77-82

23. Hackenbrack N, Rogers MB, Ashley RE et al (2017) Evolution and cryo-electron microscopy capsid structure of a North American bat Adenovirus and its relationship to other Mastadenoviruses. J Virol 91:1504-1516

24. Kim DY, Mitchell MA, Bauer RW et al (2002) An outbreak of adenoviral infection in inland bearded dragons (Pogona vitticeps) coinfected with dependovirus and coccidial protozoa (Isospora sp.). J Vet Diagn Invest 14:332-334

25. Himmel L, O'connor M, Premanandan C (2014) Necrotizing hepatitis in a domestic pigeon (Columba livia). Vet Pathol 51:1171-1173

26. Li H, Wang J, Qiu L et al (2016) Fowl adenovirus species C serotype 4 is attributed to the emergence of hepatitis-hydropericardium syndrome in chickens in China. Infect Genet Evol $45: 230-241$

27. Weber J, Stich HF (1969) Electron microscopy of cells infected with adenovirus type 2. J Virol 3:198-204

28. Mcferran JB (1998) Adenoviruses. In: Swayne DE, John R, Jackwood MW et al (eds) A laboratory manual for the isolation and identification of avian pathogens. University of Pennslyvania, Kennnet Square, pp 100-105 